\title{
Análisis del proceso de deforestación en el estado de Michoacán: de lo espacial a lo social
}

\author{
Analysis of deforestation in Michoacan state: \\ from the spatial to social
}

\author{
Jaime Octavio Loya Carrillo* \\ Jean Francois Mass Caussel ${ }^{* *}$
}

Recibido 27 de octubre de 2019; aceptado 05 de noviembre de 2019

\section{Resumen}

En el estado de Michoacán, México, se desarrollan principalmente actividades productivas en los sectores primario y secundario, esta dinámica ha favorecido la intensificación en el uso del terreno y agudizado la deforestación. En este trabajo, se plantea una propuesta metodológica que permite explicar el proceso de deforestación mediante el uso de herramientas estadísticas aplicadas en gabinete y métodos descriptivos, a partir de la observación en campo. Los resultados señalan que en ocasiones factores biofísicos, sociales, económicos y de política pública, favorecen el desmonte de los bosques y en otras, su conservación. Esta premisa puede ser la base para identificar regiones con necesidades específicas y plantear objetivos, planes de acción y/o recomendaciones, para atenderlas.

Palabras clave: deforestación, Sistemas de Información Geográfica, modelado espacial, regresión geográficamente ponderada, causas de la deforestación.

\footnotetext{
Abstract

In the Mexican state of Michoacan are developed productive activities mainly in the primary and secondary sectors, this situation has favored the intensification in the

* Universidad Nacional Autónoma de México (UNAM), México, correo electrónico: jaimeloyac@gmail.com. ORCID: https://orcid.org/0000-0002-0760-8540.

** UNAM, México, correo electrónico: fmas@ciga.unam.mx. ORCID: https://orcid.org/0000-0002-6138-9879.
} 
land use and aggravated the deforestation. In this work, a methodological proposal is used to explain the process of deforestation through the use of statistical tools and descriptive methods, based on fieldwork. The results indicate that sometimes policy public or causes biophysical, social and economic can be favored the clearing of forest and sometimes, their conservation. This premise can be the basis to identify regions with specific need and propose recommendations to address them.

Key words: deforestation, Geographic Information Systems, spatial modeling, geographically weighted regression, drivers.

\section{Introducción}

La actividad humana tiene implicaciones importantes para el Cambio Global, los cuales son controlados en gran parte por la forma en que utilizamos el suelo y los cambios que provocamos sobre la vegetación u otro material que cubre la superficie terrestre, es decir el cambio de cobertura y uso de suelo (CCUS) (Vitousek, 1992; Meyer y Turner, 1994; Lambin, 1997; Dolman et al., 2003; Rockström et al., 2009). Cuando un cambio en la cubierta vegetal se presenta, los procesos que predominan son la deforestación y la degradación forestal (Geist y Lambin, 2001; Walker, 2004), por ello la importancia de entender ¿Cuáles son las principales causas humanas del cambio de la cubierta vegetal en diferentes contextos geográficos e históricos? (NRC, 2014).

La deforestación implica la conversión de los bosques a otro tipo de uso de la tierra, causada y mantenida por alguna perturbación humana o natural (Organización de las Naciones Unidas para la Alimentación y la Agricultura — FAO, 2010). Los efectos de la deforestación son diversos y varían en todas las escalas, al igual que las causas que los promueven. Geist y Lambin (2002) clasifican las causas en dos grandes grupos: las próximas y las subyacentes. Las primeras tienen que ver con la extensión de la infraestructura, la expansión agrícola, la explotación forestal. Las segundas, con causas demográficas, económicas, tecnológicas, institucionales o políticas y culturales, así como factores ambientales, agentes biofísicos y detonantes sociales. Los cambios en la cubierta vegetal constituyen un fenómeno complejo que resulta de la interacción sinérgica de una gran cantidad de factores, de tal forma que para poder entender cómo es que este opera, es necesario un análisis sistemático a distintas escalas espaciales y temporales, que permita descubrir los principales proceso que los gobiernan (Lambien et al., 2003), En este sentido se presenta una propuesta metodológica que facilite el análisis estadístico y descriptivo entre las relaciones espaciales del proceso de deforestación con las causas que lo provocan. 


\section{Objetivos}

El objetivo general de este trabajo consistió en identificar las principales causas de la deforestación en el estado de Michoacán. A partir del objetivo general, se desglosan los siguientes objetivos particulares:

- Identificar focos de deforestación y reforestación en el estado de Michoacán, para dos cortes históricos: 2004-2007 y 2007-2014.

- Desarrollar un modelo para identificar la relación de la deforestación con los posibles factores del cambio.

- Seleccionar un caso de estudio concreto para analizar a nivel local, los patrones encontrados y explicar su relación con los factores asociados.

\section{Área de estudio}

El estado de Michoacán se localiza en la parte centro-oeste de México, la mayor parte de la superficie del estado se encuentra sobre dos provincias fisiográficas: la Sierra Madre del Sur y el Eje Neovolcánico Transversal. Las alturas oscilan desde el nivel del mar hasta cerca de los $4000 \mathrm{msnm}$. Su extensión territorial abarca aproximadamente $58 \mathrm{mil} \mathrm{km}^{2}$, lo que equivale al 3\% del territorio nacional (Figura 1).

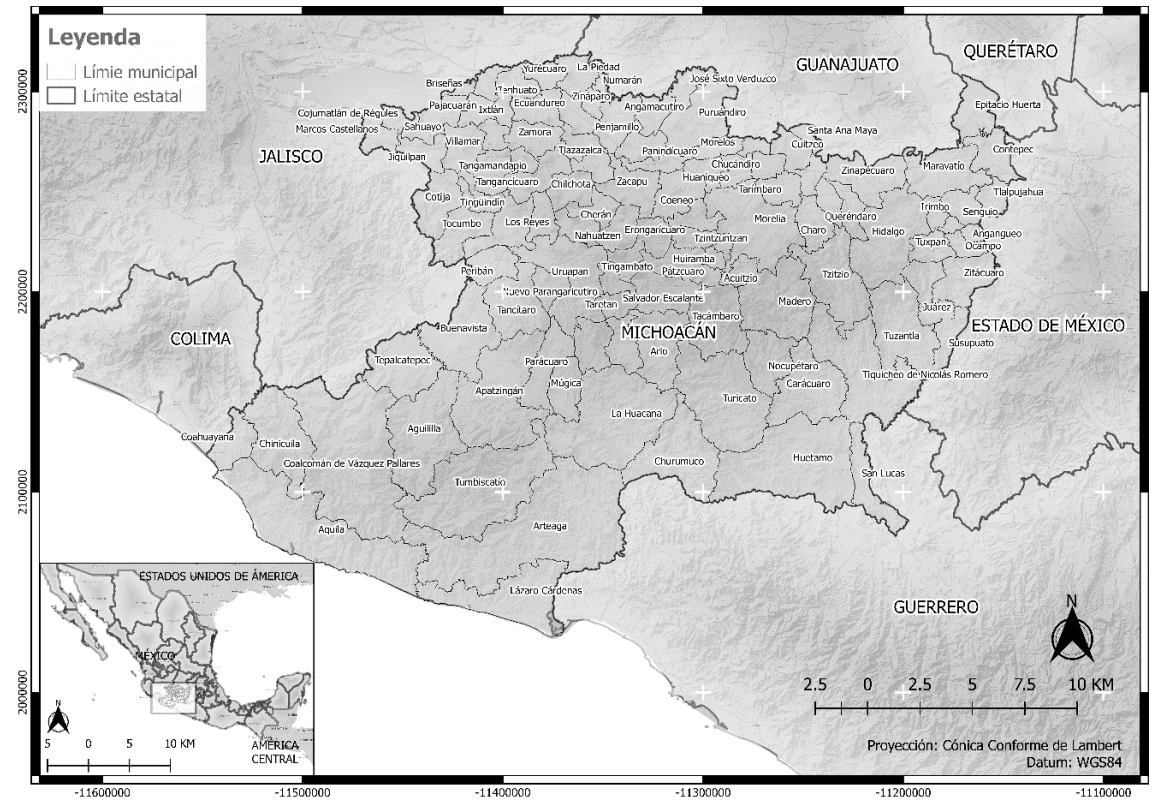

Figura 1. Localización del área de estudio. 
En Michoacán, se desarrollan actividades de los sectores primario, secundario y terciario, las cuales contribuyen con el 11.05, 23.19 y 65.76 por ciento del Producto Interno Bruto (PIB) estatal, respectivamente. En el sector primario destacan la agricultura de maíz, sorgo, aguacate, trigo y caña de azúcar, el aprovechamiento forestal y la producción pecuaria. En el sector secundario actividades de los subsectores mineros, de construcción y electricidad, mientras que el sector terciario se caracteriza por el desarrollo de actividades como: el comercio, la hotelería, el transporte, servicios bancarios, de almacenamiento y comunicación (Mercado y Palmenrín, 2012). Todos los elementos biofísicos, demográficos y económicos que se conjugan dentro del territorio michoacano, interactúan en un ambiente sinérgico para dar origen a una gran cantidad de procesos, sin embargo, en lo que a este trabajo refiere, únicamente se aborda el proceso de deforestación y su relación con los distintos factores asociados, ya que al menos hasta la década de los noventas, Michoacán presentaba una de las tasas de deforestación más elevadas a nivel nacional, e inclusive de América Latina (Bocco et al., 2001).

\section{Materiales y métodos}

En este trabajo se utilizaron Sistemas de Información Geográfica (SIG) de acceso libre QGIS (QGIS Development Team, 2015) y librerías que permiten la manipulación de datos geoespaciales (GDAL, GWMODEL, SPGWR). El análisis estadístico se llevó a cabo en el lenguaje de programación R (R Core Team, 2015). Se utilizaron los mapas de cubiertas y usos de suelo (CUS) a escala 1:50 000, elaborados por Mass et al. (2015). Dichos mapas corresponden a los años 2004, 2007 y 2014, y contienen categorías como: usos agrícolas, asentamientos humanos, distintos tipos de bosques y selvas, cuerpos de agua, manglares, y en menor medida, comunidades vegetales de papal y tular, así como zonas de vegetación aparente (compuestas por suelo desnudo, bancos de material e infraestructura). Los mapas originales se convirtieron a formato raster con una resolución espacial de 100 metros, lo cual implicó asignar la categoría que predominaba en la superficie correspondiente al pixel. Utilizar una estructura de datos raster, permitió representar las categorías de CUS de una forma más compacta, con valores de 0 para la superficie no forestal y 1 para la superficie forestal.

Posteriormente se estimaron las pérdidas y ganancias brutas, y el cambio neto en la superficie forestal. Los índices utilizados para describir el proceso de pérdidas y ganancia forestal a nivel estatal fueron los siguientes:

- Cambio neto relativo: el cambio neto es la diferencia de la superficie forestal en $t_{1}$ y en $t_{2}$, normalizado por la superficie forestal en $t_{1}$.

- Pérdida bruta relativa: superficie forestal en la primera fecha $\left(\mathrm{t}_{1}\right)$ que pasó a superficie no forestal en la fecha $2\left(t_{2}\right)$, normalizado por la superficie total de bosque en el $t_{1}$, se expresa en porcentaje $(\%)$. 
- Ganancia bruta relativa: superficie no forestal $t_{1}$ que se convirtió en superficie forestal durante $t_{2}$, normalizado por la superficie forestal en $t_{1}$, se expresa en porcentaje (\%).

Después se utilizó el estadístico Gi, el cual permitió identificar agrupaciones espaciales donde los indicadores de cambio (cambio neto, pérdida bruta y ganancia bruta) se encuentran fuera del promedio global, ya sea de forma inferior o superior, que corresponden con puntos fríos (cold spots) o puntos calientes (hot spots), respectivamente. Las agrupaciones que resultaron estadísticamente significativas, representan los patrones espaciales que podemos esperar no provengan del azar. Debido a la cantidad de tiempo necesaria para llevar a cabo este análisis, fue necesario remuestrear los archivos raster a una resolución espacial de $1 \mathrm{~km} 2$ por pixel. Para una correcta aplicación del estadístico de Gestis-Ord, es necesario especificar una distancia que permita considerar al menos 30 vecinos, cuando el número de observaciones es lo suficientemente grande. En este trabajo se utilizó una distancia de $10 \mathrm{~km}$, la cual permitió seleccionar los 100 vecinos más cercanos.

El estadístico de Gi, evalúa el promedio local entre un contexto de entidades vecinas y posteriormente, lo compara contra el promedio del conjunto total de observaciones (Getis y Ord, 1992). Para ello, es necesario establecer un nivel de confianza normalmente de 90, 95 o 99 por ciento, posteriormente para cada nivel de confianza, existe una probabilidad (Tabla 1) de que las variaciones de los patrones observados provengan del azar. En el siguiente paso se estima un valor estandarizado z o z-score, que se calcula a partir de las ecuaciones 2.1, 2.2 y 2.3. Los valores de $\mathrm{z}$ representan la desviación estándar entre las cuales, podríamos esperar la probabilidad de que la variación local del fenómeno observado provenga del azar. Los rangos de desviación estándar, se definen con el nivel de confianza. Cuando se obtienen valores de $\mathrm{z}$ altos bajos (negativos) en conjunto con probabilidades pequeñas, se rechaza la hipótesis nula de aleatoriedad al ser poco probable que le fenómeno observado provenga del azar (Figura 2).

El siguiente paso consistió en calibrar un modelo de regresión geográficamente ponderada (GWR, por sus siglas en inglés). Para ello, se seleccionaron un conjunto de variables independientes mediante el método expuesto por Lu et al. (2014). En el cual, primero se reclasificó el conjunto total de AGEB en dos categorías: deforestado o no deforestado. En este caso, se asignó un valor de 0 cuando en un AGEB la pérdida de bosque registrada fuera inferior al 2.5 por ciento respecto a la superficie original y un valor de 1, cuando la pérdida fuera superior a dicho umbral. El porcentaje de pérdida de la cubierta forestal, es un umbral que no oculta el proceso de deforestación antes de agregar los datos a nivel de AGEB. Si el umbral es mayor, entonces la mayoría de los AGEB aparecen sin deforestación. El análisis de regresión consistió de tres etapas: 
Tabla 1

Rango de valores $\mathrm{z}$ y valor de probabilidad, de acuerdo con el nivel de confianza seleccionado

\begin{tabular}{lcc}
\hline Valores de $\mathrm{z}$ & Valor $\mathrm{p}$ (probabilidad) & Nivel de confianza \\
\hline$<-1.65$ o $>1.65$ & 0.1 & $90 \%$ \\
$<-1.96 \mathrm{o}>1.96$ & 0.05 & $95 \%$ \\
$<-2.58 \mathrm{o}>2.58$ & 0.01 & $99 \%$ \\
\hline
\end{tabular}

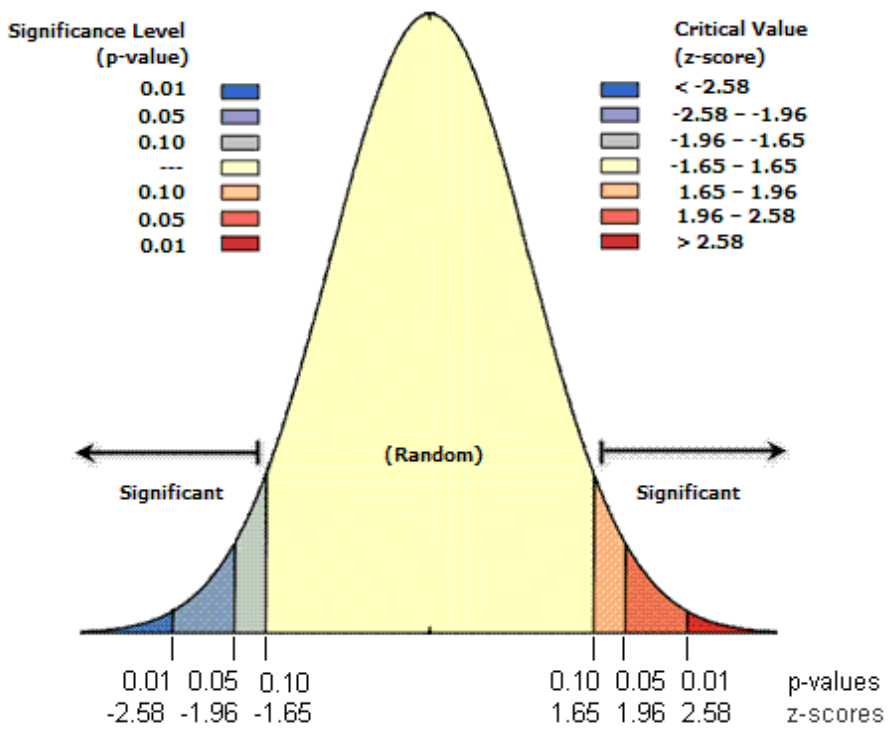

Figura 2. Gráfico de distribución de probabilidad normal estándar, entre los rangos positivos o negativos de valores $\mathrm{z}$.

Fuente: Mitchell (2005).

- Etapa 1. Selección de variables explicativas: a partir de revisión bibliográfica, conocimiento experto y disponibilidad de información espacial, se seleccionaron 32 variables potencialmente explicativas, disponibles en los portales de Internet de organismos públicos e instituciones académicas en México, tal es el caso de: el Registro Agrario Nacional (RAN), el Consejo Nacional de Población (CONAPO), la Comisión Nacional de Áreas Naturales Protegidas (CONANP), la Unidad de Informática para las Ciencias Atmosféricas y Ambientales (UNIATMOS) y el Instituto Nacional de Estadística y Geografia (INEGI) (Tabla 2). 
Tabla 2

Variables utilizadas para explicar el proceso de deforestación en el estado de Michoacán durante los periodos 2004-2007 y 2007-2017

\begin{tabular}{|c|c|c|}
\hline Variable & Descripción & Fuente \\
\hline \multicolumn{3}{|c|}{ Demográficas } \\
\hline V1 & Relación de dependencia ((POB8+POB24)/POB12) & INEGI $^{1}$ \\
\hline V2 & Porcentaje de población de 65 años y más (POB24/POB1) & INEGI \\
\hline V3 & Índice de masculinidad (POB57/POB31) & INEG| \\
\hline V4 & $\begin{array}{l}\text { Porcentaje de población de } 5 \text { años y más residente en otra entidad en junio de } \\
2005 \text { (MIG15/POB18) }\end{array}$ & INEGI \\
\hline V5 & $\begin{array}{l}\text { Porcentaje de población de } 5 \text { años y más residente en Estados } \\
\text { Unidos de América en junio de } 2005 \text { (MIG15/POB18) }\end{array}$ & INEGI \\
\hline V6 & $\begin{array}{l}\text { Porcentaje de población de } 5 \text { años y más que habla alguna lengua indígena } \\
\text { (IND10/POB18) }\end{array}$ & INEGI \\
\hline V7 & Porcentaje de hogares censales indígenas (IND19/HOGAR1) & INEGI \\
\hline V8 & Porcentaje de población de 15 años y más analfabeta (EDU28/(POB12+POB24)) & INEGI \\
\hline V9 & $\begin{array}{l}\text { Porcentaje de población sin derechohabiencia a servicios de salud } \\
\text { (SALUD2/POB1) }\end{array}$ & INEGI \\
\hline V10 & Porcentaje de hogares censales con jefatura femenina (HOGAR2/HOGAR1) & INEGI \\
\hline V11 & Porcentaje de viviendas particulares deshabitadas ((VIV0-VIV2)/HOGAR1) & INEGI \\
\hline V12 & $\begin{array}{l}\text { Porcentaje de viviendas particulares habitadas que disponen de internet } \\
\text { (VIV28/VIV2) }\end{array}$ & INEGI \\
\hline \multicolumn{3}{|c|}{ Económicas } \\
\hline V13 & Porcentaje de población ocupada (ECO4/ECO1) & \\
\hline V14 & Porcentaje de población femenina ocupada (ECO5/ECO1) & \\
\hline V15 & Mujeres ocupadas por cada 100 hombres (ECO5/ECO6) & \\
\hline V16 & Porcentaje de población desocupada (ECO25/ECO1) & \\
\hline \multicolumn{3}{|c|}{ 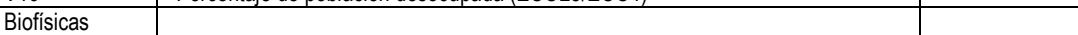 } \\
\hline DEM & Elevación (msnm) & INEGI \\
\hline PRECIPIT & Mediana de la precipitación media anual por AGEB & \\
\hline SLOPE & Mediana del porcentaje de pendiente por AGEB & INEGI \\
\hline TEMPMEDIA & $\begin{array}{l}\text { Mediana de la temperatura promedia anual registrada durante el periodo 1902- } \\
2011 \text { por AGEB }\end{array}$ & $\begin{array}{c}\text { Fernández - } \\
\text { Eguiarte et al., } \\
(2016) \text {. }\end{array}$ \\
\hline \multicolumn{3}{|c|}{ Proximidad } \\
\hline DISTLOCRUR & $\begin{array}{l}\text { Mediana de la distancia euclidiana al conjunto total de localidades rurales por } \\
\text { AGEB }\end{array}$ & INEGI \\
\hline AHRDIST & $\begin{array}{l}\text { Mediana de la distancia euclidiana a cada polígono de los } \\
\text { asentamientos humanos rurales por AGEB }\end{array}$ & INEGI \\
\hline AHUDIST & $\begin{array}{l}\text { Mediana de la distancia euclidiana a cada polígono de los } \\
\text { asentamientos humanos urbanos por AGEB }\end{array}$ & INEGI \\
\hline REDVIAL & Mediana de la distancia a la red de caminos por AGEB & \\
\hline POBTOT05 & Mediana de Población total a nivel localidad durante el año 2005 & INEGI \\
\hline \multicolumn{3}{|c|}{ Tenencia de la tierra } \\
\hline EJIDOSAH & Superficie ejidal total por AGEB destinada para asentamientos humanos & $\mathrm{RAN}^{2}$ \\
\hline EJIDOSUC & Superficie ejidal total por AGEB destinada para uso común & RAN \\
\hline EJIDOSZP & Superficie ejidal total por AGEB parcelada para uso agrícola & RAN \\
\hline PROPEJIDAL & Superficie ejidal total por AGEB & RAN \\
\hline PROPFUERA & Superficie total no ejidal por AGEB & $\begin{array}{l}\text { Elaboración } \\
\text { propia. }\end{array}$ \\
\hline \multicolumn{3}{|c|}{ Política pública } \\
\hline ANP & Superficie total por AGEB correspondiente a las áreas naturales protegidas & CONANP $^{3}$ \\
\hline ANPFUERA & Superficie total por AGEB fuera del área natural protegida & $\begin{array}{l}\text { Elaboración } \\
\text { propia }\end{array}$ \\
\hline
\end{tabular}


La unidad de análisis utilizada, disponible y de mayor detalle fueron las Áreas Geoestadísticas Básicas (AGEB) (INEGI, 2010). Se redujo el número de variables mediante un análisis de correlación global de Spearman. Primero se seleccionaron las variables menos correlacionadas entre sí, descartando variables con un coeficiente de correlación superior a 0.8 , en un segundo paso se seleccionaron las variables independientes que contaban con una correlación superior a 0.2 con la tasa de deforestación y de nuevo, se repitió el primer paso, aunque en esta ocasión se utilizó un umbral de 0.6 para descartar variables.

- Etapa 2. Análisis de multicolinealidad: Cuando existe multicolinealidad entre variables, los coeficientes de regresión se vuelven sensibles ante ligeros cambios en los datos, lo cual incrementa su varianza y los hace inestables, por esa razón se utilizaron los criterios de Belsley y Welsch (1980), quienes señala que existen problemas de multicolinealidad cuando las correlaciones locales absolutas son superiores a 0.8 (Spearman $>0.8)$, el valor de inflación de la varianza (VIF) sea mayor a 10 (VIF $>10)$, la proporción de descomposición de la varianza (VDP) mayor a $0.5(\mathrm{VDP}>0.5)$ y el número de condición local $(\mathrm{CN})$ mayor a $30(\mathrm{CN}>$ 30). Además se consideró el criterio de información de Akaike (AIC), el cual permitió definir entre todos los modelos posibles, el que mejor se ajusta a los datos, es decir, se hizo un balance entre el ajuste del modelo y el número de variables.

- Etapa 3. Ajuste del modelo GWR: Con el conjunto de variables seleccionadas, se ajustó un modelo de regresión mediante el software $\mathrm{R}$ y las librerías GWmodel y spgwr. La técnica de modelación que se utilizó en este trabajo, es referida en la literatura como GWLR (Geographically Weighted Logistic Regression), la cual explora la relación entre una variable dependiente cualitativa y dicotómica, contra un conjunto de variables independientes cualitativas o cuantitativas (Fotheringham et al., 2002; Zhang \& Gu., 2013; Rodrigues et al., 2014). Para poder estimar las estadísticas locales, es necesario definir un ancho de banda (BW, por sus siglas en inglés), el cual funciona como una ventana móvil que agrupa el número de observaciones establecidas (para más detalle consultar Fotherinhgam et al., 2002). El modelo GWLR tiene como base una ecuación de regresión logística global:

Por lo tanto la ecuación GWLR se expresa de la siguiente manera:

$$
y_{i}=\frac{e\left(\beta_{0}\left(u_{i}, v_{i}\right)+\beta_{1}\left(u_{i}, v_{i}\right) x_{1 i}+\cdots+\beta_{k}\left(u_{k i}, v_{k i}\right) x_{k i}\right)}{1+e^{\left(\beta_{0}\left(u_{i}, v_{i}\right)+\beta_{1}\left(u_{i}, v_{i}\right) x_{1 i}+\cdots+\beta_{k}\left(u_{k i}, v_{k i}\right) x_{k i}\right)}}
$$

donde $(u i, v i)$ representan las coordenadas en el espacio del punto i. De esta manera la GWL permitió obtener los coeficientes de regresión para cada ubicación en el área de estudio y posteriormente, observar la variación espacial de los coeficientes de regresión en función de un punto y sus vecinos más cercanos. 
- Etapa 4. Prueba de Monte Carlo para la significancia estadística: Monte Carlo es una prueba estadística para probar la no estacionariedad espacial, con la cual se confirma si los resultados de una GWR son estadísticamente significativos o si son artefactos de la variación aleatoria en los datos (Lu et al., 2014). Para ellos se estimó una GWR con modelos locales en la localización de cada AGEB; después a cada unidad espacial se le asignó aleatoriamente un conjunto de datos procedentes de otra observación y se calculó de nuevo una GWR, los resultados fueron comparados con los originales, este proceso se repitió 99 veces. Si los coeficientes de cada variable en el modelo GWR verdadero, se encontraron sobre la cola superior o inferior del $0.5,2.5$ o 5\% de la distribución en los datos aleatorizados, entonces puede decirse que son estadísticamente significativos con un 99, 95 y $90 \%$ de confianza respectivamente.

El análisis estadístico anterior, permitió generar mapas de correlación local de Spearman para cada AGEB, estos mapas fueron utilizados para plantear algunas hipótesis que establecen una relación positiva o negativa entre la deforestación y los distintos indicadores del cambio. Para corroborar dichas hipótesis, se realizó un estudio de caso en el cual se seleccionó al municipio de Taretan, al ser uno de los sitios con las tasas más altas de deforestación durante el periodo de análisis. Con ello, se realizaron recorridos en campo y entrevistas abiertas a distintos actores clave como: presidentes de las asociaciones cañeras, representantes del departamento de ecología del municipio, productores de guayaba, zarzamora y ganado, jefes de los Centro de Apoyo al Desarrollo Rural, además de acudir a una asamblea ejidal y entrevistar de forma personal algunos jornaleros agrícolas.

\section{Resultados}

Las tasas de deforestación para cada AGEB durante el periodo 2004 a 2007 oscilan entre el 0 y el 40 por ciento (Figura 3), gran parte de los cambios se registraron en la región centro de Michoacán, en los municipios de Uruapan, Ziracuaretiro, Taretan, Salvador Escalante, el norte de Ario de Rosales y hacia la región sierra costa, sobre los municipios de Aquila y Chincuilla. En el periodo 2007 a 2014 las tasas de deforestación disminuyeron entre un 0 y 12 por ciento (Figura 4).

Mediante el estadístico Gi, se identificaron las zonas donde los indicadores de cambio se encuentran fuera del promedio global, ya sea de forma superior o inferior. En las Figuras 5 a 10, se observan los mapas comparativos de los indicadores de cambio durante los periodos 2004-2007 y 2007-2014. Las zonas en color rojo representan los valores más altos de los distintos indicadores, mientras que los colores verdes los valores más bajos. Cabe señalar que se detectaron un gran número de AGEB sin cambios o con cambios mínimos, por lo que el valor de Gi fue de cero o próximo a cero, en los mapas aparece con tonalidades de verde claro. 


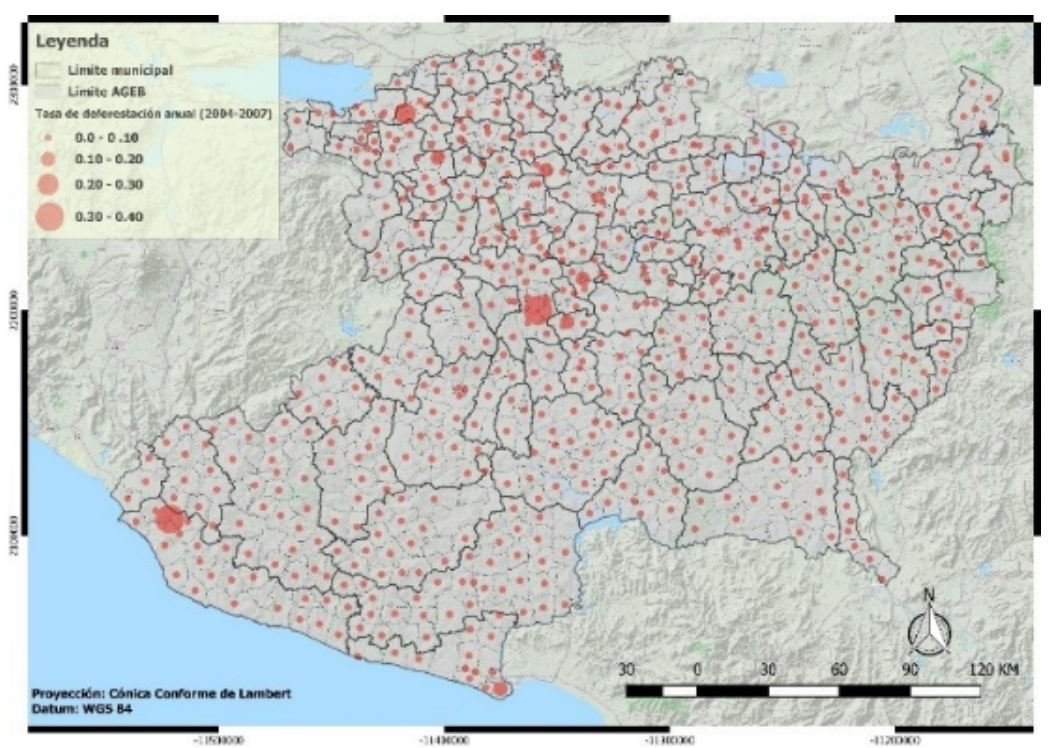

Figura 3. Tasas de deforestación por AGEB en el estado de Michoacán durante el periodo 2004-2007.

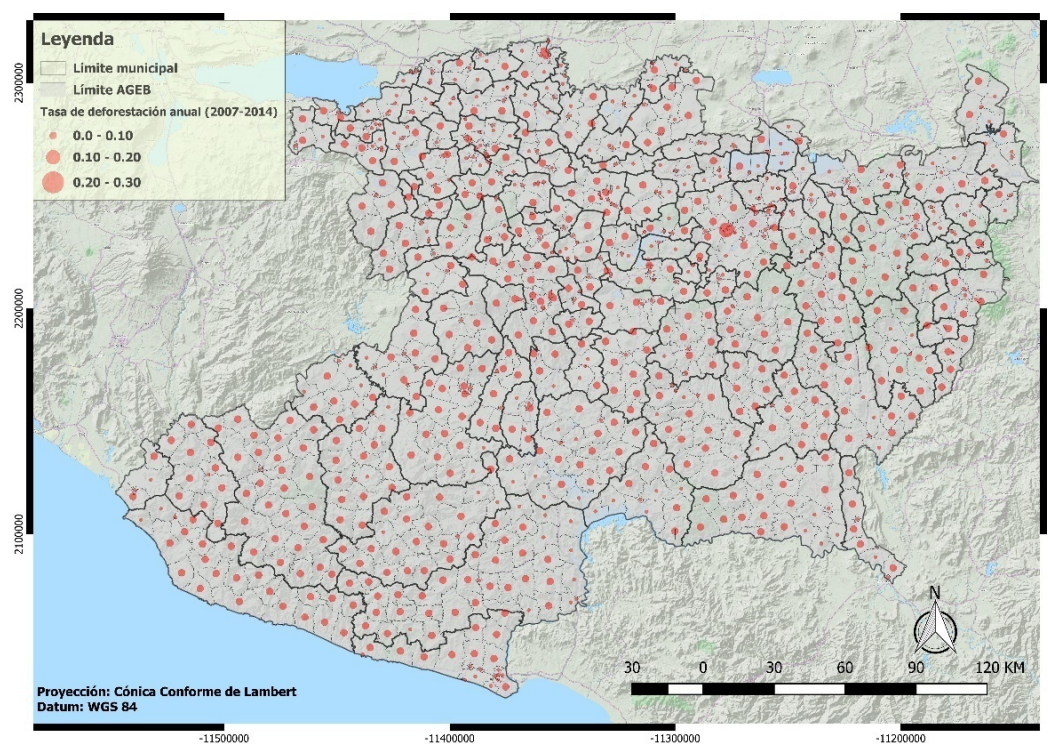

Figura 4. Tasas de deforestación por AGEB en el estado de Michoacán durante el periodo 2007-2014. 
Focos de cambio neto anual (2004-2007)

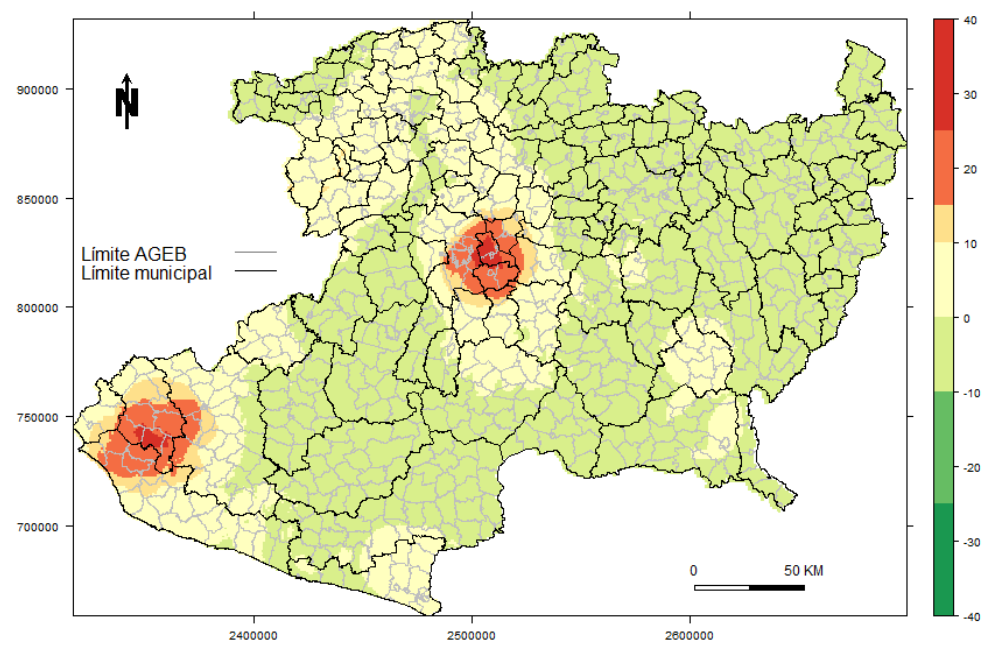

Figura 5. Mapa resultante del estadístico Gi, que representa las superficies en las cuales se concentra el mayor y menor cambio neto anual durante el periodo 2004-2007.

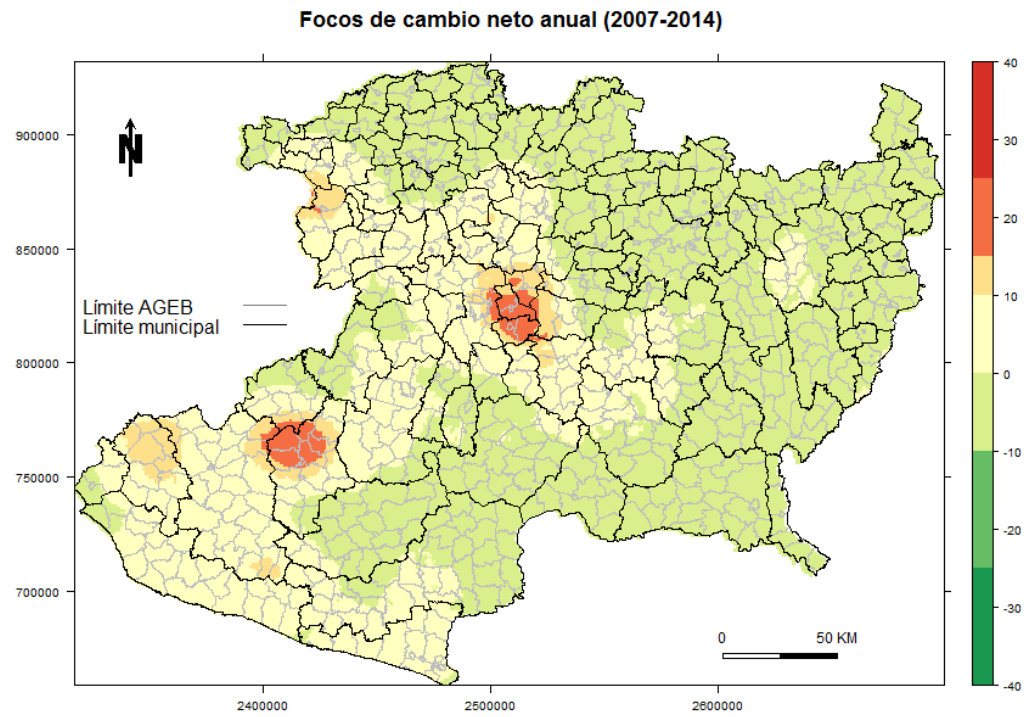

Figura 6. Mapa resultante del estadístico Gi, que representa las superficies en las cuales se concentra el mayor y menor cambio neto anual durante el período 2007-2014. 
Focos de deforestación bruta (2004-2007)

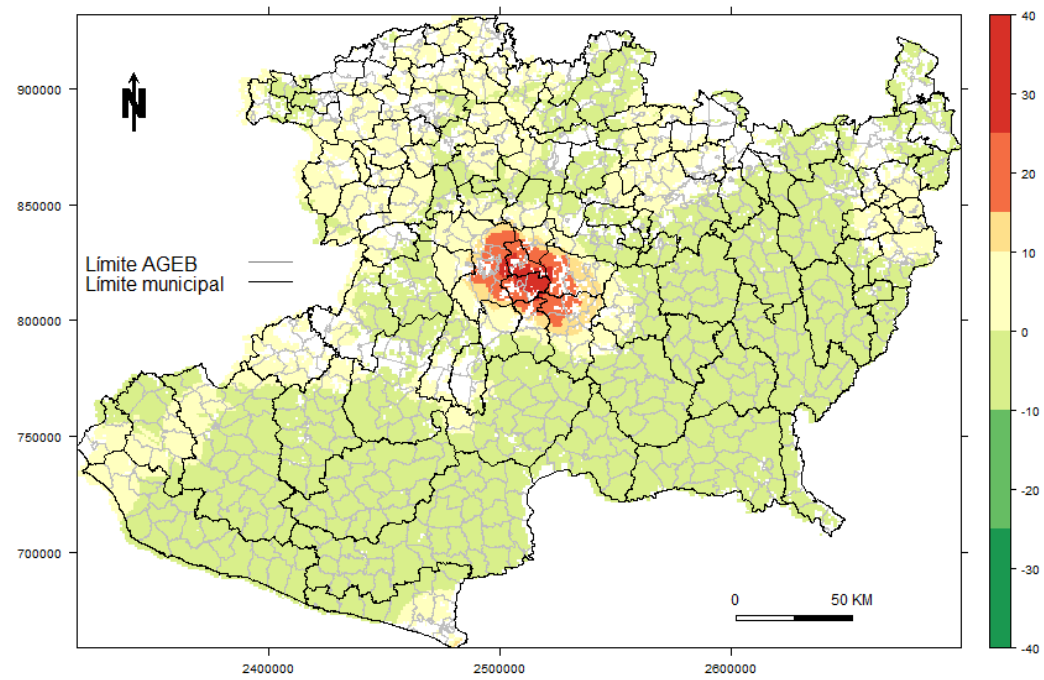

Figura 7. Mapa resultante del estadístico Gi, que representa las superficies con mayor y menor pérdida bruta relativa durante el período 2004-2007.

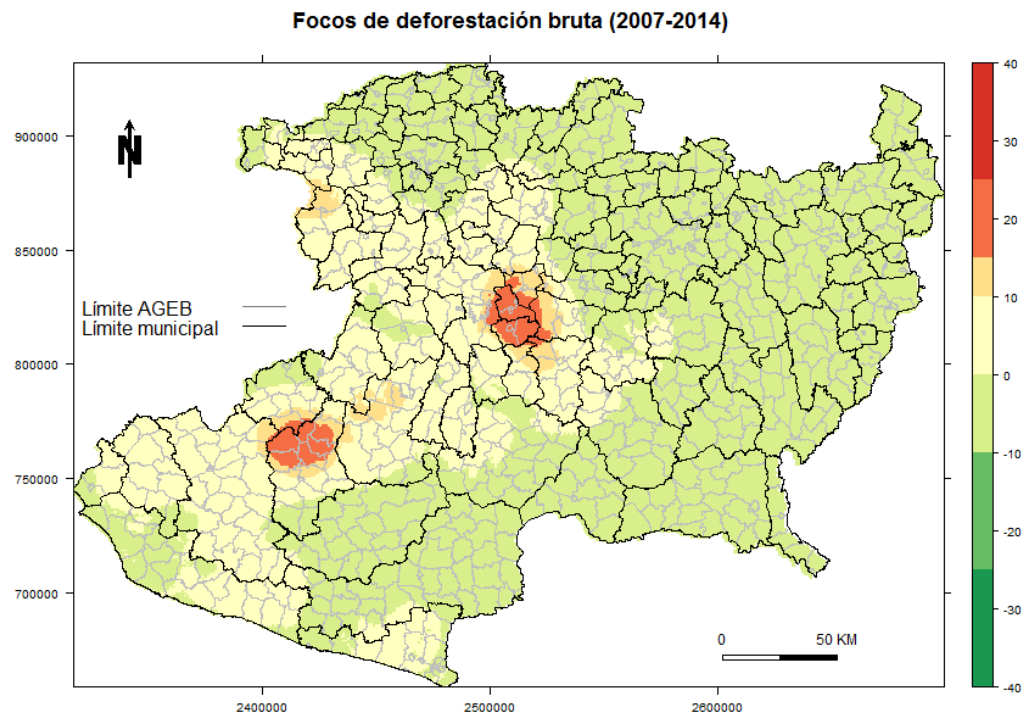

Figura 8. Mapa resultante del estadístico Gi, que representa las superficies con mayor y menor pérdida bruta durante el periodo 2007-2014. 


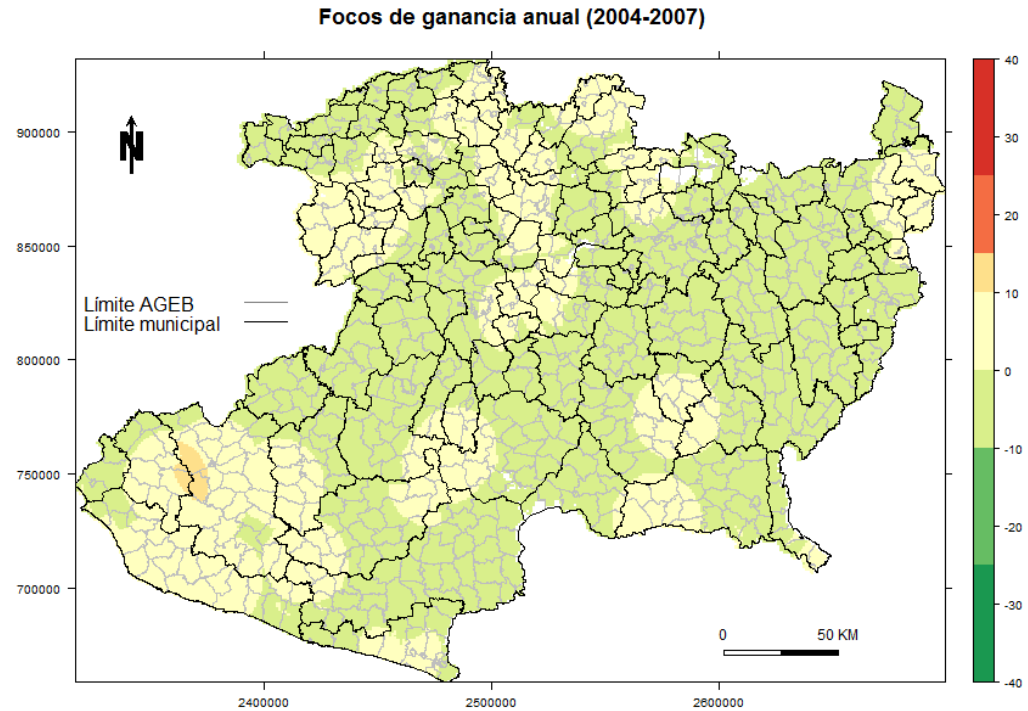

Figura 9. Mapa resultante del estadístico Gi, que representa las superficies con mayor y menor ganancia bruta relativa durante el periodo 2004-2007.

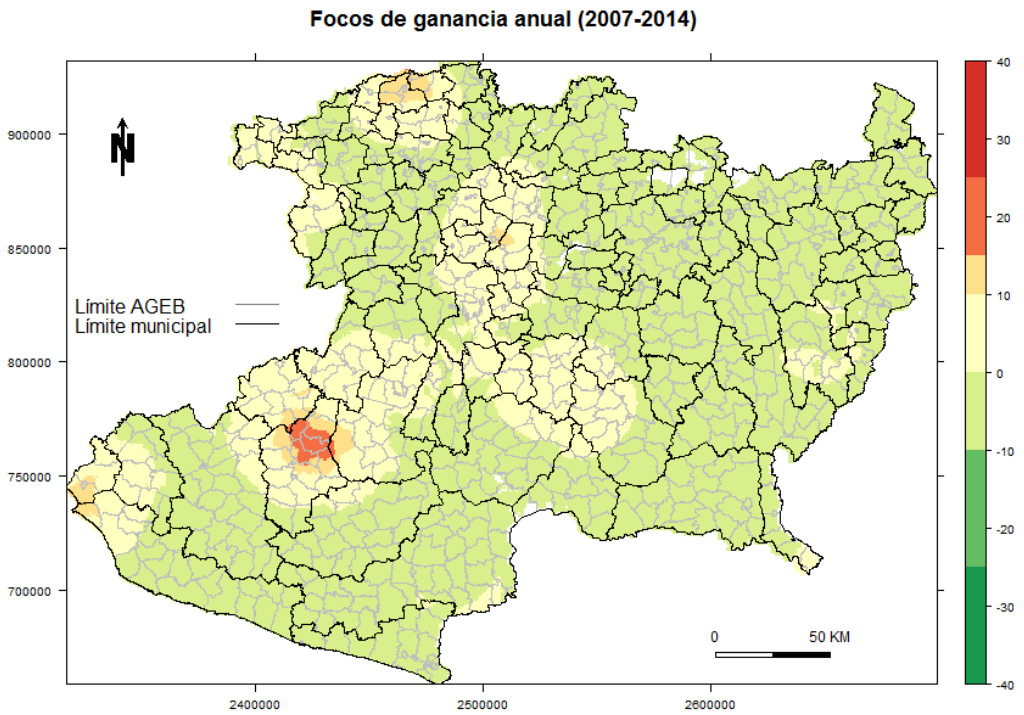

Figura 10. Mapa resultante del estadístico Gi, que representa las superficies con mayor y menor ganancia bruta relativa durante el periodo 2007-2014. 
Los principales focos de deforestación (Figuras 7 y 8 ) se encuentran fuertemente relacionados con actividades agrícolas. En la región centro, se favorecen los cultivos de exportación (como el aguacate). En la región sudoeste se observan pérdidas y ganancias forestales, relacionadas con actividades agrícolas y pecuarias, así como aprovechamientos forestales para la producción de resina y la industria maderera, mientras que el proceso de ganancia forestal, se favorece por presencia de programas de reforestación y restauración con altos niveles de eficacia. Por otro lado, en el municipio de Taretan son varias las causas que promueven la deforestación, sin embargo, durante la investigación de campo se identificó la interacción entre factores económicos, sociales y ambientales, que en algunas ocasiones son favorecidas por instrumentos gubernamentales. En la Tabla 3, se resumen cuáles son los factores que promueven la deforestación.

\section{Discusión y conclusiones}

Gracias al uso de métodos directos e indirectos que utilizan distintos enfoques de modelación espacial, se ha mejorado considerablemente el entendimiento del CCUS (Agarwal et al., 2002; NRC, 2014). Aunado a esto, el avance tecnológico y la apertura a distintas fuentes de datos e información, ha permitido integrar métodos estadísticos, de aprendizaje automático y minería de datos, que mejoran con bastante precisión las estimaciones respecto al uso de los bosques (Austin et al., 2009; Garcia et al., 2019; NRC, 2014). Los métodos utilizados en este trabajo nos han permitido comprobar que la agricultura que produce para los mercados internacionales, es la principal causa de deforestación en el estado de Michoacán, al igual que ha ocurrido en varios países del mundo, principalmente de América Latina (Chaves y Carvalho, 2019; De Sy et al., 2019; Geist y Lambin, 2002). El trabajo de otros autores en el estado de Michoacán, señala que particularmente el proceso de deforestación ha sido motivado por el estímulo económico que favorece el desmonte de los bosques, para cultivar aguacate de exportación y/o convertirlo en huertos frutícolas de mayor valor económico (Morales y Cuevas, 2011; Chávez-León et al., 2012; Morales et al., 2012). Mientras que los remanentes de bosque, son degradados por la interacción de la tala ilegal, la extracción de madera para combustible, el sobrepastoreo y los incendios forestales (Sáenz, 2005).

Merino (2005) documentó el efecto de las políticas públicas (forestal, agraria y de conservación) en dos comunidades de la Reserva de la Biosfera Mariposa Monarca (RBMM) en el estado de Michoacán e identificó, un impacto diferencial, propiciado por las distintas condiciones que privan el uso de los bosques. Aunque esta autora utilizó escalas de análisis y perspectivas distintas a la de nuestro trabajo, sus resultados señalan que el efecto de una política pública se expresa de forma distinta sobre el territorio, ya que en algunas ocasiones favorece la conservación de los bosques y en otras, el deterioro. Bajo este contexto, los indicadores socioeconómicos, biofísicos 
Tabla 3

Factores que promueven la deforestación en el municipio de Taretan

\begin{tabular}{|c|c|c|c|}
\hline \multicolumn{2}{|c|}{ Factores } & Variables & Impactos \\
\hline \multicolumn{2}{|l|}{ 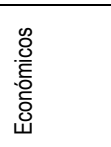 } & $\begin{array}{l}\text { Creación de pequeña y medianas } \\
\text { empresas } \\
\text { Incremento de ingresos } \\
\text { Desarrollo de infraestructura } \\
\text { Accesibilidad }\end{array}$ & $\begin{array}{l}\text { Generación de empleos } \\
\text { Expropiación de los recursos } \\
\text { Economía extractivista }\end{array}$ \\
\hline \multicolumn{2}{|l|}{$\begin{array}{l}\frac{\mathscr{\omega}}{\frac{\pi}{0}} \\
\frac{\pi}{0}\end{array}$} & $\begin{array}{l}\text { Dinámicas demográficas } \\
\text { Incorporación de la mujer a la fuerza } \\
\text { laboral (principalmente para el cultivo } \\
\text { de zarzamora) } \\
\text { Nivel educativo } \\
\text { Población indígena } \\
\text { Expansión de los asentamientos } \\
\text { humanos } \\
\text { Extracción de madera } \\
\text { Arraigo }\end{array}$ & $\begin{array}{l}\text { Retención de jóvenes y adultos } \\
\text { Identidad social } \\
\text { Desintegración familiar } \\
\text { Violencia y conflictos armados }\end{array}$ \\
\hline \multirow[t]{2}{*}{ 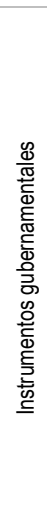 } & 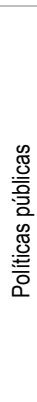 & $\begin{array}{l}\text { PROAGRO } \\
\text { PROGRAN } \\
\text { Diésel agropecuario } \\
\text { PIMAF } \\
\text { Fomento a la agricultura } \\
\text { (modernización de } \\
\text { maquinaria y equipo) } \\
\text { Proyectos estratégicos } \\
\text { Apoyo a invernaderos } \\
\text { Sistemas de riego }\end{array}$ & \multirow[t]{2}{*}{$\begin{array}{l}\text { Acceso a servicios de salud } \\
\text { Norma las actividades agrícolas en todo el proceso del cultivo } \\
\text { Asistencia técnica } \\
\text { Subsidios a través de incentivos (fertilizantes, insecticidas y } \\
\text { productos orgánicos) } \\
\text { Acceso a tecnologías eficientes (tractores y aspersores) } \\
\text { Reconversión productiva } \\
\text { Seguro agrícola } \\
\text { Apoyo a pequeños productores (maíz y frijol principalmente) } \\
\text { Incremento en la productividad agrícola y pecuaria } \\
\text { Desarrollo tecnológico } \\
\text { Investigación } \\
\text { Incremento en el uso de hidrocarburos } \\
\text { Favorecen la extracción de los recursos naturales }\end{array}$} \\
\hline & ঐ & $\begin{array}{l}\text { Ley de Desarrollo Sustentable de la } \\
\text { Caña de Azúcar (LDSCA) }\end{array}$ & \\
\hline \multicolumn{2}{|l|}{ 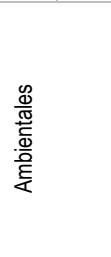 } & $\begin{array}{l}\text { Precipitación } \\
\text { Temperatura } \\
\text { Elevación } \\
\text { Edafología } \\
\text { Recursos hídricos } \\
\text { Incendios forestales }\end{array}$ & $\begin{array}{l}\text { Condiciones climáticas adecuadas para la siembra de algunos } \\
\text { cultivos } \\
\text { Degradación de suelos } \\
\text { Desabasto y deterioro de los recursos hídricos } \\
\text { Extracción de recursos naturales } \\
\text { Incremento de la contaminación por el uso de fertilizantes e } \\
\text { hidrocarburos } \\
\text { Expansión de los usos agrícolas a costa de los bosques y selvas } \\
\text { Degradación de la cubierta forestal }\end{array}$ \\
\hline
\end{tabular}

y de política pública que utilizamos para explicar el proceso de deforestación, también se expresan de forma diferenciada sobre el territorio. Por ello, resulta interesante comparar las conclusiones anteriores con los resultados de este trabajo, ya que ambos contribuyen con distintos elementos para entender la no estacionariedad espacial en el CCUS y la deforestación. 
La ventaja de utilizar métodos de análisis cuantitativos o cualitativos locales, es la posibilidad de identificar regiones con necesidades específicas y con ello, proponer objetivos, planes de acción o recomendaciones para atenderlas. Este trabajo aporta evidencias para comprender las causas que operan detrás del proceso de deforestación en el estado de Michoacán. Es una propuesta metodológica que integra el uso de herramientas estadísticas aplicadas en gabinete y métodos descriptivos, a partir de la observación en campo.

En algunas ocasiones, el efecto de las instituciones no se refleja directamente sobre el proceso de deforestación, sin embargo detona actividades como: la reconversión productiva en el sector agrícola, garantiza el acceso a recursos tecnológicos en zonas con potencial productivo y de mercado, modifica las dinámicas poblacionales (fenómenos de migración, nivel educativo, incorporación de la mujer al mercado laboral), propicia el desarrollo de pequeñas y medianas empresas, lo cual desde luego, impacta sobre el uso de los recursos naturales (no solo los bosques). Las instituciones públicas son un mediador importante en el proceso de toma de decisiones, ya que inciden sobre la autonomía de las comunidades locales. Es difícil hablar de gobernanza forestal cuando las decisiones respecto al uso de los recursos atienden a presiones de mercado, demográficas o de política pública. No es posible consolidar una buena gobernanza forestal cuando existen cuestiones como: pobreza, bajos niveles de educación y seguridad social, desigualdad o intereses económicos de ciertos grupos de poder. Los retos sobre la gobernanza forestal se centran en el diseño de políticas públicas, la legitimización en el uso de los recursos y la voluntad de los organismos operadores, para tomar decisiones informadas y consensadas respecto al uso de los bosques.

\section{Bibliografía}

Agarwal, C., Green, G.M., Grove, J.M., Evans, T.P., y Schweik, C.M. (2002). “A review and assessment of land-use change models: dynamics of space, time, and human choice", Gen. Tech. Rep. NE-297. Newton Square, PA: US Department of Agriculture, Forest Service, Northeastern Research Station. 61.

Austin, K.G., Schwantes, A., Gu, Y. and Kasibhatla, P.S. (2019). "What causes deforestation in Indonesia?", Environmental Research Letters, 14(2), 024007.

Belsley, D.A. and Kuh, E. Welsch., R.E. (1980). Regression Diagnostics: Identifying Influential Data and Sources of Collinearity, Uiley Series in Probability and Mathematical Statistics.

Bocco, G., Mendoza, M. y Masera, O.R. (2001). "La dinámica del cambio del uso del suelo en Michoacán: Una propuesta metodológica para el estudio de los procesos de deforestación", Investigaciones geográficas, 44: 18-36.

Chaves, M.E.D. y de Carvalho Alves, M. (2019). "Recent applications of the MODIS sensor for soybean crop monitoring and deforestation detection in Mato Grosso, Brazil", CAB Reviews, 14(007): 1-9. 
Chávez León, G., Tapia Vargas, L.M., Bravo Espinoza, M., Sáenz Reyes, J., Muñoz Flores, H.J., Vidales Fernández, I., Larios Guzmán, A., Rentería Ánima J.B., Villaseñor Ramírez, F.J., Sánchez Pérez, J. de la Luz., Alcantar Rocillo, J.J. y Mendoza Cantú, M. (2012). Impacto de cambio de uso de suelo forestal a huertos de aguacate.

De Sy, V., Herold, M., Achard, F., Avitabile, V., Baccini, A., Carter, S., Clevers, J.G. P.W., Lindquist, E., Pereira, M. y Verchot, L. (2019)." Tropical deforestation drivers and associated carbon emission factors derived from remote sensing data", Environmental Research Letters, 14(9), 094022.

Dolman, A.J., Verhagen, A. and y Rovers, C.A. (2003). Global environmental change and land use, Springer Science \& Business Media.

FAO (Organización de las Naciones Unidas para la Agricultura y la Alimentación). (2010). Evaluación de los recursos forestales mundiales 2010. Términos y definiciones.

Fotheringham, A.S., Brunsdon, C. and Charlton, M. (2002). "Geographically weighted regression: the analysis of spatially varying relationships", John Wiley \& Sons.

Garcia, A.S., de FN Vilela, V.M., Rizzo, R., West, P., Gerber, J.S., Engstrom, P.M., y Ballester, M.V.R. (2019). “Assessing land use/cover dynamics and exploring drivers in the Amazon's arc of deforestation through a hierarchical, multi-scale and multi-temporal classification approach", Remote Sensing Applications: Society and Environment, 15, 100233.

Garibay, C. y Bocco, G. (2011). Cambios de uso de suelo en la meseta purépecha (1976-2005), INE-Semarnat y CIGA-UNAM, México.

Geist, H.J. y Lambin, E.F. (2002). A meta-analysis of proximate and underlying causes of deforestation based on subnational case study evidence. LUCC Report Series, 4.

Geist, H. J., y Lambin, E.F. (2002). A meta-analysis of proximate and underlying causes of deforestation based on subnational case study evidence. LUCC Report Series, 4.

Getis, A., y Ord, J. K. (1992). The analysis of spatial association by use of distance statistics. Geographical analysis, 24(3): 189-206.

INEGI (Instituto Nacional de Estadística y Geografía) (2010). Censo Nacional de Población y Vivienda 2010.

Lambin, E.F. (1997). "Modelling and monitoring land-cover change processes in tropical regions", Progress in Physical Geography, 21(3): 375-393. http://doi.org/10.1177/030913339702100303

Lambin, E.F., Geist, H.J., y Lepers, E. (2003). "Dynamics of land-use and land-cover change in tropical regions", Annual review of environment and resources, 28(1): 205-241. 
Lu, B., Harris, P., Charlton, M. y Brunsdon, C. (2014). “The GWmodel R package: further topics for exploring spatial heterogeneity using geographically weighted models", Geo-spatial Information Science, 17(2): 85-101.

Mas, J.F., González, R. Lemoine-Rodríguez, R., López-Sánchez, J., Piña-Garduño, A. y Herrera-Flores, E. "Actualizaciones sucesivas de mapas de cubierta del suelo combinando segmentación de imágenes e interpretación visual”, Memoria en extensor SELPER-XXI-México-UACJ-2015, Ciudad Juárez, Chihuahua, México, 2015.

Mercado Vargas, H. y Palmerín Cerna, M. (2012). El estado de Michoacán y sus regiones turísticas.

Merino Pérez, L. (2004). Conservación o deterioro: el impacto de las políticas públicas en las instituciones comunitarias y en los usos de los bosques en México, México, Instituto Nacional de Ecología.

Meyer, W.B., y B.L. Turner, I.I. (1994). Changes in land use and land cover: a global perspective (vol. 4), Cambridge University Press.

Mitchell, A. (2005). La Guía de Esri para el análisis SIG.

Morales, L.M. y Cuevas, G. (2011). Inventarios 1974-2007, y evaluación del impacto ambiental regional del cultivo del aguacate en el estado de Michoacán. Informe Ejecutivo, Centro de Investigaciones en Geografía Ambiental, UNAM, Michoacán, México.

Morales-Manila, L.M., A. Reyes-González, G. Cuevas-Garcia y M. Onchi-Ramuco (2012). Inventario 2011 del cultivo del aguacate en el estado de Michoacán (pp. 89). Morelia, Michoacán, Centro de Investigaciones en Geografía Ambiental, UNAM -COFUPRO, 89 pp.

National Research Council (2014). Advancing Land Change Modeling: Opportunities and Research Requirements, National Academies Press.

QGIS Development Team (2015). QGIS Geographic Information System, Open Source Geospatial Foundation Project. http://qgis.osgeo.org

R Core Team (2013). "R: A language and environment for statistical computing. $\mathrm{R}$ Foundation for Statistical Computing", Vienna, Austria. Recuperado de http://www.R-project.org/

Rockström, J., Steffen, W.L., Noone, K., Persson, Å., Chapin III, F.S., Lambin, E., Lenton, Foley, J. (2009). Planetary boundaries: exploring the safe operating space for humanity.

Rodrigues, M., de la Riva, J. y Fotheringham, S. (2014). "Modeling the spatial variation of the explanatory factors of human-caused wildfires in Spain using geographically weighted logistic regression", Applied Geography, 48: 52-63.

Songchitruksa, P. y Zeng, X. (2010). "Getis-Ord spatial statistics to identify hot spots by using incident management data", Transportation Research Record: Journal of the Transportation Research Board, 2165: 42-51. 
Vitousek, P.M. (1994). "Beyond global warming: ecology and global change", Ecology, 75(7): 1861-1876.

Walker, R. (2004). "Theorizing land-cover and land-use change: The case of tropical deforestation", International Regional Science Review, 27(3): 247-270.

Zhang, L. and Gu, Y. (eds.) (2013). Information Systems and Computing Technology, CRC Press. 